Department of Econometrics and Business Statistics

http://business.monash.edu/econometrics-and-business-

statistics/research/publications

\title{
Dimension reduction for outlier detection using DOBIN
}

Sevvandi Kandanaarachchi and Rob J Hyndman

August 2019

Working Paper 17/19 


\title{
Dimension reduction for outlier detection using DOBIN
}

\author{
Sevvandi Kandanaarachchi \\ Department of Econometrics and Business Statistics, \\ Monash University, \\ Melbourne, Australia. \\ Email: sevvandi.kandanaarachchi@monash.edu
}

\section{Rob J Hyndman}

Department of Econometrics and Business Statistics, Monash University,

Melbourne, Australia.

Email: rob.hyndman@monash.edu

22 August 2019

JEL classification: C14,C38,C88 


\section{Abstract}

This paper introduces DOBIN, a new approach to select a set of basis vectors tailored for outlier detection. DOBIN has a solid mathematical foundation and can be used as a dimension reduction tool for outlier detection tasks. We demonstrate the effectiveness of DOBIN on an extensive data repository, by comparing the performance of outlier detection methods using DOBIN and other bases. We further illustrate the utility of DOBIN as an outlier visualization tool. The R package dobin implements this basis construction.

Keywords: outlier detection, dimension reduction, outlier visualization, basis vectors

\section{Introduction}

Outlier detection is used in diverse applications such as the identification of extreme weather events, stock market crashes and fraudulent transactions. A common challenge in many domains is that a data point may be an outlier in the original high dimensional space, but not an outlier in the low dimensional subspace created by a dimension reduction method.

We address this challenge by proposing a set of basis vectors tailored for unsupervised outlier detection which we call DOBIN: a Distance based Outlier BasIs using Neighbours. The acronym DOBIN is inspired from the informal verb 'dob in', which the Collins English dictionary defines as 'to inform against or report, specially to the police'. We emphasize that DOBIN is not an outlier detection method; rather it is a pre-processing step that can be used by any outlier detection method.

To the best of our knowledge no formal dimension reduction techniques exist for outlier detection. It is common to use Principal Component Analysis (PCA) where dimension reduction is used when detecting outliers (e.g., Talagala et al., 2019; Hyndman, Wang \& Laptev, 2015). However, the goal of PCA is to find a set of basis vectors that explains the variance of the data, such that the highest variance is in the direction of the first vector, and so on. Therefore, the PC basis may not be suited for detecting outliers. By introducing DOBIN, we aim to bridge this gap in the literature.

We envisage two uses of DOBIN. First, as a basis more conducive to outlier detection, it brings outliers to the forefront using a smaller number of components. In this sense, it is somewhat similar to subspace outlier detection (e.g., Aggarwal \& Yu, 2001; Keller, Muller \& Bohm, 2012), although DOBIN does not detect outliers directly. If outliers arise in the full input space, DOBIN 
enables the outlier detection algorithm to detect outliers using fewer components. The second use of DOBIN is to assist with visualisation of outliers as we will see in Section 4.

The literature on outlier detection has evolved in two fields, with contributions from statisticians and computer scientists. Although the methodologies used by the two groups are somewhat different, both groups have contributed to a rich and diverse literature, benefiting the broader community. Useful summaries of the computer science literature are provided by Goldstein \& Uchida (2016) and Zimek, Schubert \& Kriegel (2012); while similar surveys of the statistics outlier literature are given in Rousseeuw \& Leroy (2005) and Unwin (2019a). We have included methods from both disciplines in this paper.

Some methods treat outlier identification as a binary rule, with observations classified as outliers or otherwise (e.g., Billor, Hadi \& Velleman, 2000; Wilkinson, 2017; Rousseeuw \& Bossche, 2018), while other methods rank all observations in terms of "outlyingness" (e.g., Breunig et al., 2000; Liu, Ting \& Zhou, 2008), or provide a probability of an observation being an outlier (e.g., Kriegel et al., 2009). This difference impacts the performance evaluation metrics. Metrics such as false negatives and false positives or positive predictive value and negative predictive value are used to evaluate the binary results (Wilkinson, 2017). By contrast, having a ranking of the observations leads to use tools such as area under the Receiver Operator Characteristic (ROC) curve, area under the Precision-Recall (PR) curve or precision at $n$ to evaluate performance (Campos et al., 2016).

In Sections 3 and 5 we investigate the effect of DOBIN on three outlier detection methods which first appeared in the computer science literature: LOF (Breunig et al., 2000), KNN (Ramaswamy, Rastogi \& Shim, 2000) and iForest (Liu, Ting \& Zhou, 2008). To evaluate their performance, we use area under the ROC curve. We examine the effectiveness of DOBIN by comparing it against two other bases: PCA and the original basis. In Section 3 we use synthetic data to conduct experiments and in Section 5 we use real data from our repository of more than 12,000 datasets (Kandanaarachchi et al., 2019).

Section 4 examines outlier detection methods developed by the statistical community on some associated datasets. We use O3 plots by Unwin, 2019a, which can be used to compare the results of six different outlier detection methods: HDoutliers (Wilkinson, 2017), mvBACON (Billor, Hadi \& Velleman, 2000), adjOutlyingness (Brys, Hubert \& Rousseeuw, 2005), covMcd (Rousseeuw \& Driessen, 1999) , FastPCs (Vakili \& Schmitt, 2014) and DetectDeviatingCells (Rousseeuw \& Bossche, 2018). We use O3 plots in conjunction with the first two DOBIN components of each 
dataset and visually inspect if the outliers identified by the $\mathrm{O} 3$ plots are further away from the other points in the DOBIN space. This section highlights the usage of DOBIN as an outlier visualization tool.

We have produced an R package dobin (Kandanaarachchi, 2019), which computes the DOBIN basis. In addition, all examples in this paper are available in the supplementary materials at https://github.com/sevvandi/Outlier-Basis. We start the next section with the mathematical framework of DOBIN.

\section{Mathematical Framework}

Let $X_{N \times p}$ be a matrix denoting a dataset of $N$ observations and $p$ attributes. Let us denote the $i^{\text {th }}$ row of $X$ by $x_{i}$. The distance between the observations $x_{i}$ and $x_{j}$ can be written as

$$
\operatorname{dist}\left(x_{i}, x_{j}\right)^{2}=\left(x_{i}-x_{j}\right)^{\top} S\left(x_{i}-x_{j}\right),
$$

where $S$ is a symmetric positive definite matrix. In addition when $S$ is diagonal, i.e. $S=$ $\operatorname{diag}\left(s_{1}, s_{2}, \ldots, s_{p}\right)$, we obtain

$$
\operatorname{dist}\left(x_{i}, x_{j}\right)^{2}=\left\langle\eta,\left(x_{i}-x_{j}\right) \circ\left(x_{i}-x_{j}\right)\right\rangle,
$$

where $\langle\cdot, \cdot\rangle$ denotes the standard inner product in $\mathbb{R}^{p}, \eta=\left(s_{1}, s_{2}, \ldots, s_{p}\right)^{\top}$, and $\circ$ denotes the element-wise vector product. As $S$ is symmetric and positive definite it is diagonalizable, thus giving us motivation for considering a diagonal $S$.

\subsection{The $Y$ space}

Next we establish the $Y$ space, which is instrumental in the construction of DOBIN. Let $\boldsymbol{y}_{i j}=$ $\left(x_{i}-x_{j}\right) \circ\left(x_{i}-x_{j}\right)=\left(\left(x_{i 1}-x_{j 1}\right)^{2},\left(x_{i 2}-x_{j 2}\right)^{2}, \ldots,\left(x_{i p}-x_{j p}\right)^{2}\right)$. Substituting in equation (1) gives

$$
\operatorname{dist}\left(x_{i}, x_{j}\right)^{2}=\left\langle\eta, y_{i j}\right\rangle
$$

By finding the appropriate $\eta$ of unit length, we can maximize the distance between $x_{i}$ and $\boldsymbol{x}_{j}$. This prompts the question as to which $x_{i}$ and $x_{j}$ need to be chosen to maximize the distance between them, if outlier detection is the goal. If we use knn distances as a guiding principle, we are interested in the $k$ nearest neighbours of a given point. Consequently, we can choose $x_{i}$ and $x_{j}$ that share a common neighbourhood. 
If we were to calculate $\boldsymbol{y}_{i j}$ for all pairs, it would be computationally expensive as there are $N(N+1) / 2$ pairs to consider. In addition, this would give us pairwise distances between points that we are not interested in, such as points on the boundary of the point cloud, that are opposite each other. Finding $\eta$ that maximizes the distances between such points is not beneficial for outlier detection, because a large distance between polar-opposite points does not mean that either of those points are outliers. Therefore we do not compute $\boldsymbol{y}_{i j}$ for all pairs of $\boldsymbol{x}_{i}$ and $\boldsymbol{x}_{j}$; rather we select the pairs that we want to include in our $Y$ space.

For each point $\boldsymbol{x}_{i}$ we compute $\boldsymbol{y}_{i j}$ arising from a set a nearest neighbours. From Kandanaarachchi et al., 2018 we know that nearest neighbours depend on the normalization technique. As a result, we choose two normalization techniques to pre-process the data; Min-Max and Median-IQR. Min-Max normalization scales each column to values between 0 and 1, with the minimum mapped to 0 and the maximum mapped to 1 . Median-IQR scales each column to median 0 and IQR 1. Let $X_{1}$ denote the normalized data using Min-Max and $X_{2}$ using Median-IQR. For each normalization technique we choose a set of nearest neighbours from $k_{1}$ to $k_{2}$, that is for any point $x_{i}$ we choose the $k_{1}^{\text {th }}$ nearest neighbour to the $k_{2}^{\text {th }}$ nearest neighbour. Here $k_{1}$ and $k_{2}$ are parameters and one can choose $k_{1}=1$.

Next, for each point we find the union of nearest neighbours computed using the two normalization techniques. For example, for a given point $x_{i}$, if the nearest neighbours according to Min-Max are $\left\{x_{2}, x_{5}, x_{9}\right\}$ and Median-IQR gives $\left\{x_{4}, x_{5}, x_{7}\right\}$, then we consider the union of these two sets as the set of nearest neighbours of $x_{i}$. Using the nearest neighbours we construct a list of pairs denoted by T. Continuing the same example, the pairs $\left(x_{i}, x_{2}\right),\left(x_{i}, x_{4}\right),\left(x_{i}, x_{5}\right),\left(x_{i}, x_{7}\right)$, $\left(x_{i}, x_{9}\right)$, and so on will be in $T$.

For each pair in $T$, we compute $\boldsymbol{y}_{i j}$ as in equation (1) on the normalized space $X_{1}$ or $X_{2}$, depending on user preference. We now have the initial $Y$ space: a set of $\tilde{M}$ points in $\mathbb{R}^{p+}$. As the list of pairs that give rise to the initial $Y$ space are contained in $T$, we can denote $Y=\left\{\boldsymbol{y}_{\ell}\right\}_{\ell=1}^{\tilde{M}}$, where each $\boldsymbol{y}_{\ell}=\boldsymbol{y}_{i_{(\ell)} j_{(\ell)}}$, i.e. $\ell$ is the row number in the matrix $Y$ and each $\boldsymbol{y}_{\ell}$ comes from the $\ell^{\text {th }}$ pair in $T$.

We are interested in neighbouring distances that are relatively large. The Euclidean distance squared between two points in the $X_{1}$ or $X_{2}$ space can be written as $\sum_{m=1}^{p} y_{\ell m}$ for the appropriate $\ell$. In order to consider only points with relatively large distances, we remove points that contribute to distances below a certain threshold determined by a percentile, i.e. we remove points such that $\sum_{m=1}^{p} y_{\ell m}<Q$ where $Q$ is the $q^{\text {th }}$ percentile of $\left\{\sum_{m=1}^{p} y_{\ell m}\right\}_{\ell=1}^{\tilde{M}}$. We remove the associated pairs from $T$ as well. This constitutes the $Y$ space: $Y=\left\{\boldsymbol{y}_{\ell}\right\}_{\ell=1}^{M}$. 
The main advantage of the $Y$ space is that it makes distance computation between two points in the $X$ space a linear function. This makes maximizing distances between points a much easier task. Algorithm 1 summarizes the construction of the $Y$ space.

input $\quad: X, k_{1}, k_{2} \in \mathbb{Z}^{+}, q \in(0,1)$ and choice of normalization.

output : The space $Y$ consisting of all $\left\{\boldsymbol{y}_{\ell}\right\}_{\ell=1}^{M}$ and the associated indices $i$ and $j$.

1 Normalize $X$ using Min-Max normalization. Let us call this space $X_{1}$.

2 Find $k_{1}$ to $k_{2}$ nearest neighbours for each point in $X_{1}$.

3 Next normalize $X$ using Median-IQR normalization. Let us call this space $X_{2}$.

4 Find $k_{1}$ to $k_{2}$ nearest neighbours for each point in $X_{2}$.

5 Then for each point collate all unique neighbours from both Min-Max and

Median-IQR normalization methods.

6 Let $T=$ the set of $i$ and $j$ indices, corresponding to the pairs of points in $X$.

7 For all these pairs, compute $y_{i j}$ as in equation (2) using $X_{1}$ or $X_{2}$ space according to the choice of normalization. Let $Y=\left\{\boldsymbol{y}_{1}=\boldsymbol{y}_{i_{(1)} j_{(1)}}, \boldsymbol{y}_{2}=\boldsymbol{y}_{i_{(2)} j_{(2)}}, \ldots, \boldsymbol{y}_{\tilde{M}}=\boldsymbol{y}_{i_{(\tilde{M})} j_{(\tilde{M})}}\right\}$, where $\left(i_{(1)}, j_{(1)}\right)$ is the first $(i, j)^{\text {th }}$ pair in $T$. Consequently $Y$ is an $\tilde{M} \times p$ matrix, with the $\ell^{\text {th }}$ row denoted by $\boldsymbol{y}_{\ell}$.

8 Let $Q$ be the $q^{\text {th }}$ percentile of $\left\{\sum_{m=1}^{p} y_{\ell m}\right\}$. The quantity $\sum_{m=1}^{p} y_{\ell m}$ is the distance between points $x_{i_{(\ell)}}$ and $x_{j_{(\ell)}}$ in $X_{1}$ or $X_{2}$ space.

9 Remove points for which $\sum_{m=1}^{p} y_{\ell m}<Q$.

10 Remove the associated pairs of $(i, j)$ from $T$.

11 The remaining points constitute the $Y$ space: $Y=\left\{\boldsymbol{y}_{\ell}\right\}_{\ell=1}^{M}$.

Algorithm 1: Construction of the $Y$ space.

Figure 1 illustrates the $X$ and $Y$ spaces where $X$ is a 2 dimensional normal distribution of 100 points with a single outlier depicted in red. We see that the $Y$ space consists of a lesser number of points compared to $X$, of which 6 points are contributed by the outlier. This is because in the construction of the $Y$ space 5 neighbours are considered for each point with $k_{1}=1$ to $k_{2}=5$,

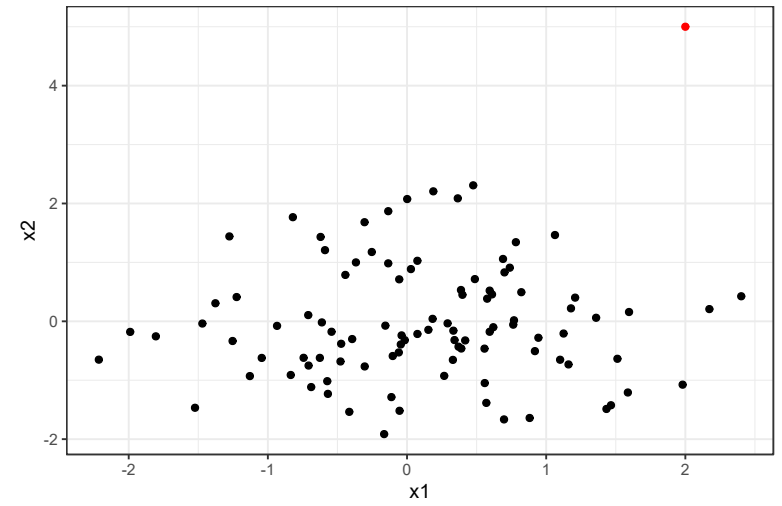

(a)

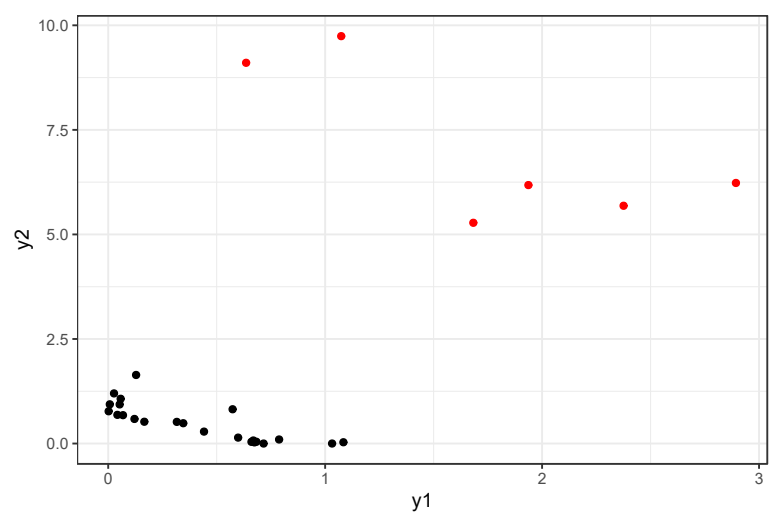

(b)

Figure 1: The $X$ and $Y$ spaces. The $X$ space in (a) and the associated $Y$ space in $(b)$ with $q=0.95$. The red coloured point in the $X$ space gives rise to 6 points, which are coloured in red in the $Y$ space. 
and the points $y_{k}$ with relatively small distances $\left(\sum_{i} y_{k i} \leq Q\right)$ are removed. Thus, the effect of outliers is magnified in the $Y$ space.

We summarise the salient features of the $Y$ space below:

1. $Y=\left\{\boldsymbol{y}_{\ell}\right\}_{\ell=1}^{M}$, where $\boldsymbol{y}_{\ell} \in \mathbb{R}^{p+}$.

2. Each $y_{\ell} \in Y$ is constructed from two points $x_{i}$ and $x_{j}$ in $X$ with $T(\ell)=(i, j)$, where $T$ is a list consisting of the pairs in $X$ that are used to construct $Y$. In addition, $x_{i}$ and $x_{j}$ are $k$-nearest neighbours for some $k \in\left\{k_{1}, \ldots, k_{2}\right\}$.

3. For $i, j, \ell$ as above, $y_{\ell}=\left(x_{i}-x_{j}\right) \circ\left(x_{i}-x_{j}\right)$, where $\circ$ denotes the element-wise vector product.

4. The $Y$ space contains points $\boldsymbol{y}_{\ell}$ with relatively high $\sum_{m=1}^{p} y_{\ell m}$, i.e. it contains vectors that contribute to high knn distances in the $X$ space.

\subsection{Maximising the distance between points}

Restating equation (2) using $T(\ell)=(i, j)$ we obtain

$$
\sum_{(i, j) \in T} \operatorname{dist}\left(x_{i}, x_{j}\right)^{2}=\sum_{\ell}\left\langle\eta, y_{\ell}\right\rangle
$$

Thus the total distance squared for relatively high nearest neighbour distances $\sum_{(i, j) \in T} \operatorname{dist}\left(\boldsymbol{x}_{i}, \boldsymbol{x}_{j}\right)^{2}$ can be maximised by finding the appropriate $\eta$ which maximises $\sum_{\ell}\left\langle\eta, \boldsymbol{y}_{\ell}\right\rangle$. However, (3) depends on the scale of $\eta$, so we normalize $\eta$ such that $\|\eta\|=1$. We now state our optimisation problem as

$$
\max _{\eta} \sum_{\ell}\left\langle\eta, \boldsymbol{y}_{\ell}\right\rangle \quad \text { subject to }\|\eta\|^{2}=1
$$

Solving this using Lagrange Multipliers we obtain $\nabla f=\lambda \nabla g$, with

$$
f(\eta)=\sum_{\ell}\left\langle\eta, y_{\ell}\right\rangle \quad \text { and } \quad g(\eta)=\|\eta\|^{2}-1=0
$$

As $\nabla f=\left(\frac{\partial f}{\partial \eta_{1}}, \frac{\partial f}{\partial \eta_{2}}, \cdots, \frac{\partial f}{\partial \eta_{p}}\right)$ we compute

$$
\frac{\partial f}{\partial \eta_{j}}=\frac{\partial}{\partial \eta_{j}} \sum_{\ell}\left\langle\eta, \boldsymbol{y}_{\ell}\right\rangle=\frac{\partial}{\partial \eta_{j}} \sum_{\ell=1}^{M} \sum_{i=1}^{p} \eta_{i} y_{\ell i}=\sum_{\ell=1}^{M} \sum_{i=1}^{p} \delta_{i j} y_{\ell i}=\sum_{\ell=1}^{M} y_{\ell j}
$$


where $\delta_{i j}$ is the Kronecker delta function which equals 1 when $i=j$ and zero otherwise. Hence

$$
\nabla f=\left(\sum_{\ell=1}^{M} y_{\ell 1}, \sum_{\ell=1}^{M} y_{\ell 2}, \ldots, \sum_{\ell=1}^{M} y_{\ell p}\right)=\sum_{\ell=1}^{M} \boldsymbol{y}_{\ell}
$$

Similarly, as $g(\eta)=\left(\sum_{j=1}^{p} \eta_{j}^{2}\right)-1$, we get $\nabla g=\eta$. By substituting $\nabla f=\lambda \nabla g$ and using $\|\eta\|=1$, we obtain

$$
\eta=\frac{\sum_{\ell=1}^{M} \boldsymbol{y}_{\ell}}{\left\|\sum_{\ell=1}^{M} \boldsymbol{y}_{\ell}\right\|}
$$

\subsection{Constructing a basis}

We have computed the vector $\eta$ that maximises $\sum_{\ell}\left\langle\eta, \boldsymbol{y}_{\ell}\right\rangle$. To find a basis, we need to find the second, third and subsequent vectors which are orthogonal and in some way maximise the quantity $\sum_{\ell}\left\langle\eta, \boldsymbol{y}_{\ell}\right\rangle$. Let us first rename $\eta$ as $\eta_{1}$. Taking $\eta_{1}$ as the first basis vector, we take the projection of vector $\boldsymbol{y}_{\ell}$ on to $\eta_{1}$ and remove these components from $\boldsymbol{y}_{\ell}$ :

$$
\boldsymbol{y}_{\ell_{1}}=\boldsymbol{y}_{\ell}-\left\langle\eta_{1}, \boldsymbol{y}_{\ell}\right\rangle \eta_{1}
$$

Thus $\boldsymbol{y}_{\ell_{1}} \perp \eta_{1}$. Let $Y_{1}=\left\{\boldsymbol{y}_{\ell_{1}}\right\}_{\ell=1}^{M}$ be the set of remaining components of $\left\{\boldsymbol{y}_{\ell}\right\}_{\ell=1}^{M}$ after removing the projection on to $\eta_{1}$. Now we can compute $\eta_{2}$ which maximises $\sum_{\ell}\left\langle\eta, \boldsymbol{y}_{\ell_{1}}\right\rangle$ as

$$
\eta_{2}=\frac{\sum_{\ell=1}^{M} \boldsymbol{y}_{\ell_{1}}}{\left\|\sum_{\ell=1}^{M} \boldsymbol{y}_{\ell_{1}}\right\|}
$$

Proceeding in this way we obtain

$$
\begin{aligned}
\boldsymbol{y}_{\ell_{b}} & =\boldsymbol{y}_{\ell_{b-1}}-\left\langle\eta_{b}, \boldsymbol{y}_{\ell_{b-1}}\right\rangle \eta_{b}, \\
\text { and } \quad \eta_{b+1} & =\frac{\sum_{\ell=1}^{M} \boldsymbol{y}_{\ell_{b}}}{\left\|\sum_{\ell=1}^{M} \boldsymbol{y}_{\ell_{b}}\right\|},
\end{aligned}
$$

with $\boldsymbol{y}_{\ell_{0}}=\boldsymbol{y}_{\ell}$. The set $\Theta=\left\{\eta_{1}, \eta_{2}, \ldots, \eta_{p}\right\}$ gives a basis for $Y$ with each $\eta_{i+1}$ maximising $\sum_{\ell}\left\langle\eta, \boldsymbol{y}_{\ell_{i}}\right\rangle$. This process is somewhat similar to Gram Schmidt orthogonalisation.

Note that Equation (3) is only true for $\boldsymbol{y}_{\ell} \in \mathbb{R}^{p+}$. The other $Y$ spaces $\left(Y_{1}, Y_{2}, \ldots\right)$ are not subsets of $\mathbb{R}^{p+}$. To adjust for this, after computing $\boldsymbol{y}_{\ell_{b}}$ as in equation (6), we temporarily change the basis such that the points $\left\{\boldsymbol{y}_{\ell_{b}}\right\}_{\ell=1}^{M}$ are in a positive orthant. Then after computing $\eta_{b+1}$ as in equation (7) using positive $\left\{\boldsymbol{y}_{\ell_{b}}\right\}_{\ell=1}^{M}$, we transform it back again to the original basis. 
Once the basis $\Theta$ is computed, we change the basis of the original data as follows:

$$
\tilde{X}=X_{1} \Theta \quad \text { or } \quad \tilde{X}=X_{2} \Theta
$$

where $X_{1}$ or $X_{2}$ is the normalized $X$ space according to the choice of normalization. We note that the basis $\Theta$ is not invariant to rotations; i.e. if one were to start with a different basis, the resulting $\Theta$ would be different. This is because when constructing the $Y$ space we compute element-wise squares of vector differences. While vector addition is basis invariant, squared element-wise components depend on the basis vectors. Notwithstanding this limitation, we achieve good results for experiments conducted on our extensive collection of more than 12,000 real world datasets. For almost all of our experiments, we constructed DOBIN using the original basis as input. We only used a different basis if the number of variables were much higher than the number of observations. Furthermore, as we will see in Section 4 it is easier to gain insights about the outlying directions when the axes are original variables.

We conclude this mathematical discussion by summarizing the key steps of DOBIN:

1. Find the $Y$ space for a given dataset $X$ as detailed in Algorithm 1.

2. Construct the basis $\Theta$ as outlined in Section 2.3.

3. Transform the original space $X$ using equation (8).

\subsection{Parameters of DOBIN}

We have three numerical parameters $k_{1}, k_{2}$ and $q$ for constructing the $Y$ space with the following default values:

$$
\begin{aligned}
k_{2} & =\min (20, \max (\lfloor N / 20\rfloor, 2)), \\
k_{1} & =\max \left(k_{2}-10,1\right), \\
q & =0.95,
\end{aligned}
$$

where $N$ is the number of observations in the dataset. The parameters $k_{1}$ and $k_{2}$ affect the run time of the algorithm, so we set a maximum value of 20 for $k_{2}$. As $k_{1}<k_{2}$, we consider a maximum of 10 neighbours for each point. The parameter $q$ determines the cut-off quantile needed to obtain a subset of $y$ with large distances. Therefore $q$ needs to be a relatively high value in the interval $(0,1)$. From equation (4) we maximise the projection of $y$ on to $\eta$. If $q$ is 
too low, many $y$ with smaller projections may contribute heavily to the sum, making DOBIN vectors less effective.

The other parameter for constructing the $Y$ space is the choice of normalization. While Min-Max normalization is commonly used by the computer science community (Campos et al., 2016), other normalization techniques such as distance from univariate medians and Mahalanobis distance are commonly used by the statistical community (Billor, Hadi \& Velleman, 2000). We give two options for normalization: Min-Max and Median-IQR.

The purpose of the final parameter is to aid visualisation in two dimensions. As points $\left\{\boldsymbol{y}_{\ell}\right\}_{i=1}^{M}$ lie in the positive orthant, the first vector $\eta_{1}$ which maximizes $\sum_{\ell}\left\langle\eta \boldsymbol{y}_{\ell}\right\rangle$ also lies in the positive orthant. However, this may not render the best possible 2D-visualization of the outliers in $X$ space, because points can have positive and negative coordinates in X space. Hence we compute the signed $\boldsymbol{y}_{\ell}$, denoted by $\tilde{\boldsymbol{y}}_{\ell}$, by keeping track of the signs of the point differences $\left(\boldsymbol{x}_{i}-\boldsymbol{x}_{j}\right)$. Let $T(\ell)=(i, j)$, then

$$
\tilde{y}_{\ell}=\operatorname{sign}\left(x_{i}-x_{j}\right) \circ y_{\ell}
$$

where $\circ$ denotes element-wise multiplication. Then

$$
\begin{aligned}
& \operatorname{sign}\left(\eta_{1}\right)=\operatorname{sign}\left(\sum_{i=1}^{M} \tilde{y}_{\ell}\right), \\
& \text { and } \quad \tilde{\eta}_{1}=\operatorname{sign}\left(\eta_{1}\right) \circ \eta_{1},
\end{aligned}
$$

where $\eta_{1}$ is computed using equation (5). However, this adds to the computation and is only recommended if 2D-visualization is an aim of the exercise.

\section{Experiments with synthetic data}

In this section we do three experiments with synthetic data. For each experiment we compare results for three outlier detection methods, namely LOF (Breunig et al., 2000), KNN (Ramaswamy, Rastogi \& Shim, 2000) and isolation forest (Liu, Ting \& Zhou, 2008). We choose these methods as they are well recognised and fundamentally different: LOF uses a local density based approach; KNN uses a global distance based approach; and iForest uses a randomised tree based approach. For each outlier detection method we consider the following three coordinate systems:

1. All variables in the dataset; 
2. Perform Principal Component Analysis (PCA) on the dataset, and use the first half of the principal components (PCs).

3. Perform DOBIN on the dataset, and use the first half of the DOBIN components.

For each outlier detection method, we compare the results using these three sets of coordinates. We use area under the Receiver Operator Characteristic curve (AUC) as our performance measure.

\subsection{Experiment 1}

The first experiment considers two normal distributions: an inlier normal distribution and an outlier normal distribution, which moves out from the inlier distribution as its mean increases. We consider a dataset of 405 observations in $\mathbb{R}^{6}$, of which 400 observations in each dimension are normally distributed with mean 0 and standard deviation 1 . The remaining 5 observations signify outliers and are normally distributed with mean $\mu$ and standard deviation 0.2 in one dimension, and mean 0 and standard deviation 1 in other dimensions. The value of $\mu$ is changed from 0 to 4.5 by increments of 0.5 . The reason for a smaller standard deviation in the first dimension is to ensure that the outliers stay close to the mean $\mu$ and move out of the inlier distribution as $\mu$ increases. For each value of $\mu$ we consider the three sets of coordinates described above and their performance using the outlier methods LOF, KNN and iForest. We perform 10 iterations of this experiment. Figure 2 shows the results for all three outlier methods using the average performance values of 10 iterations.

Using 3 DOBIN components gives the highest performance for all 3 outlier detection methods for $\mu>1$. Indeed, we are interested in a set of coordinates that can accentuate the outliers as they move out from the inlier distribution. For values of $\mu \in\{0,0.5,1\}$, the outlier distribution lies in the interior of the inlier distribution, making a performance comparison between the coordinates not meaningful.

\subsection{Experiment 2}

The second experiment considers a bi-modal setting with two inlier normal distributions and one outlier normal distribution which moves into the valley as its mean changes. Somewhat similar to the previous example we consider 805 observations in $\mathbb{R}^{6} ; 800$ inlier observations of which 400 centred at $(5,0,0,0,0,0)$ and the other 400 centred at $(-5,0,0,0,0,0)$. All inlier observations come from normal distributions with standard deviation 1 in each dimension. The 
Area under ROC for LOF, KNN and iForest
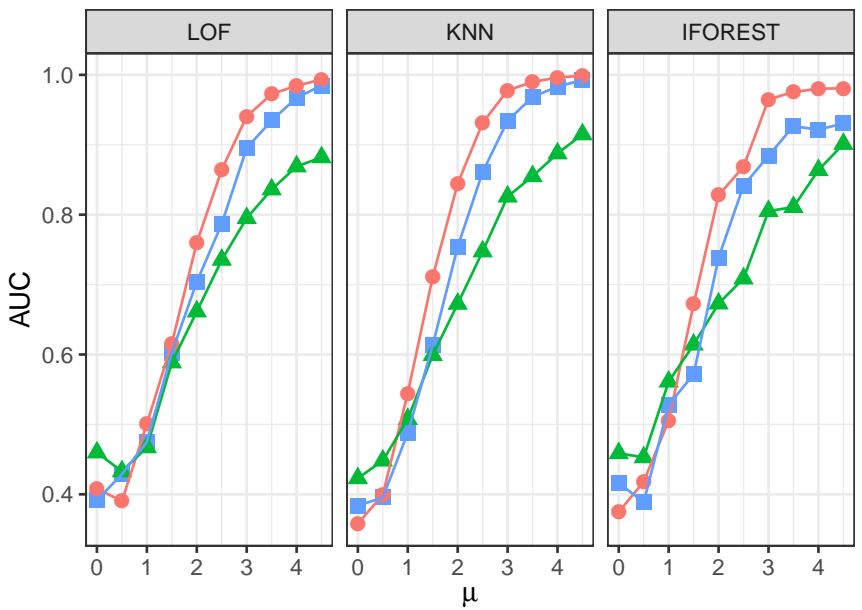

\section{Coordinates}

- 3 DOBIN comps

- 3 PC comps

늘 All Vars

Figure 2: Results of Experiment 1, in $\mathbb{R}^{6}$.

Area under ROC for LOF, KNN and iForest
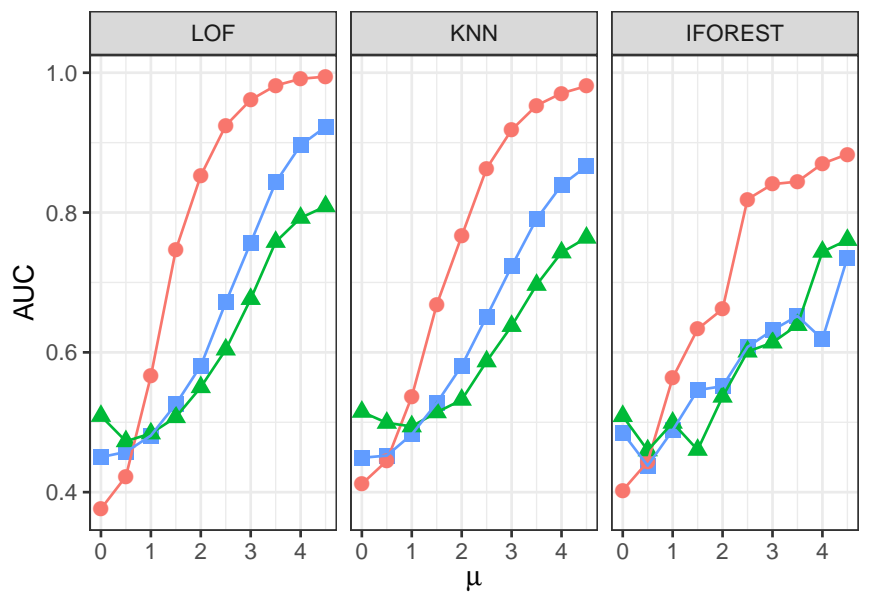

Coordinates

- 3 DOBIN comps

- 3 PC comps

플 All Vars

Figure 3: Results of Experiment 2, in $\mathbb{R}^{6}$.

Area under ROC for LOF, KNN and iForest
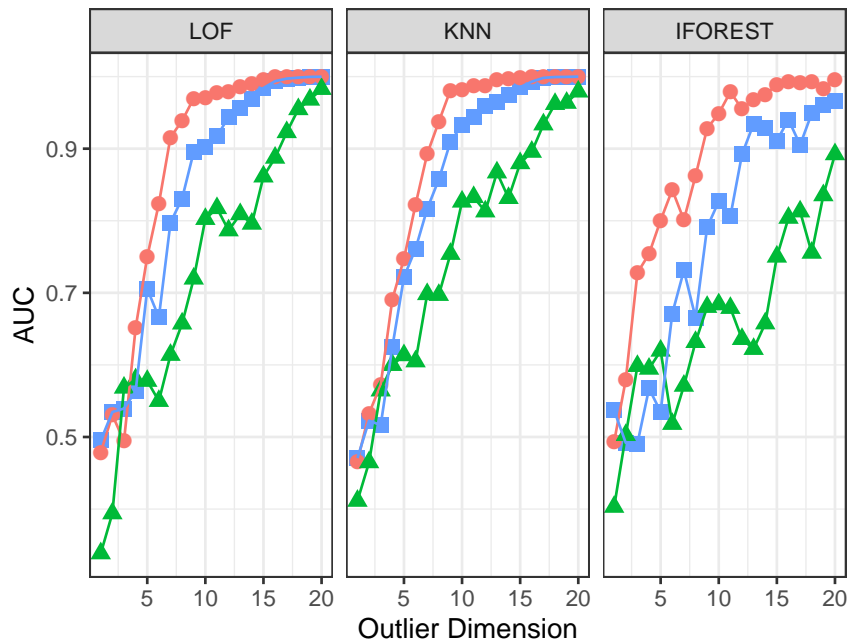

Coordinates

- 10 DOBIN comps

- 10 PC comps

- All Vars

Figure 4: Results of Experiment 3, in $\mathbb{R}^{20}$. 
outlier distribution consists of 5 points with mean $(5-\mu, 0,0,0,0,0)$ and standard deviations 0.2 in the first dimension and 1 in other dimensions. The value of $\mu$ is increased from 0 to 4.5 in increments of 0.5. Again, a smaller standard deviation is to ensure that the outliers move into the valley. Figure 3 shows the results for all three outlier methods on all three coordinate systems using the average values of 10 iterations.

Again we see that using 3 DOBIN components gives the highest performance for all three outlier methods.

\subsection{Experiment 3}

The third experiment is motivated from Zimek, Schubert \& Kriegel, 2012. We consider a uniform distribution in $\mathbb{R}^{20}$ and place a manual outlier at 0.9 in $i$ dimensions where $i$ is changed from 1 to 20 . While this is not an outlier in any single dimension, as $i$ increases this point stands out from the rest of the distribution. Indeed, for large $i$ this point lies furthest from the rest. An equivalent outlier is 0.1 in $i$ dimensions. The reason that these points are outliers is because they are at the corners of the hyper-cube and so are far away from other points. On the contrary, 0.5 in $i$ dimensions would be closest to other points. This is because $(0.5,0.5, \ldots)$ has neighbours from all sides and has on average $2^{20}$ more neighbours compared to $(1,1, \ldots)$ which is a corner on the unit hyper-cube. For practical purposes the point $(0.9,0.9, \ldots)$ is similar to $(1,1, \ldots)$ and has significantly less neighbours compared to $(0.5,0.5, \ldots)$. Figure 4 shows the results of all three outlier methods on all three coordinate systems. Again we see that using half of the DOBIN components gives the best performance for all three outlier methods.

In all three experiments we see that DOBIN coordinates give the best performance followed by the full set of coordinates. Principal Components are generally less effective than the full set of coordinates for these three experiments and outlier methods. We note that this is not a failure of PCA, as outlier detection is not its intended goal. Rather, PCA maximizes the variance in each component such that a low dimensional representation of the dataset is a good approximation to the original. These results suggest that DOBIN is preferred over PCA as a dimension reduction technique for outlier detection.

\section{Results with visualization}

In this section we investigate specific examples motivated from Unwin, 2019a and Wilkinson, 2017 using O3 plots and the DC1-DC2 space, which is the space spanned by the first two DOBIN 
vectors. O3 plots (Unwin, 2019a) can be used to compare the results of 6 different outlier detection methods: HDoutliers, mvBACON, adjOutlyingness, covMcd, FastPCs, and DetectDeviatingCells. Besides facilitating an ensemble of outlier methods, O3 plots also highlight outliers in axis parallel subspaces. We use O3 plots as a means of validating the DC1-DC2 space.

\subsection{Election2005 dataset}

We start with the Election2005 dataset (Grimm, 2019), which is used by Unwin, 2019a to illustrate the $\mathrm{O} 3$ plot. This dataset includes election results of two German elections and certain attributes of the electorates. We examine the O3 plot and the DC1-DC2 space of this dataset.

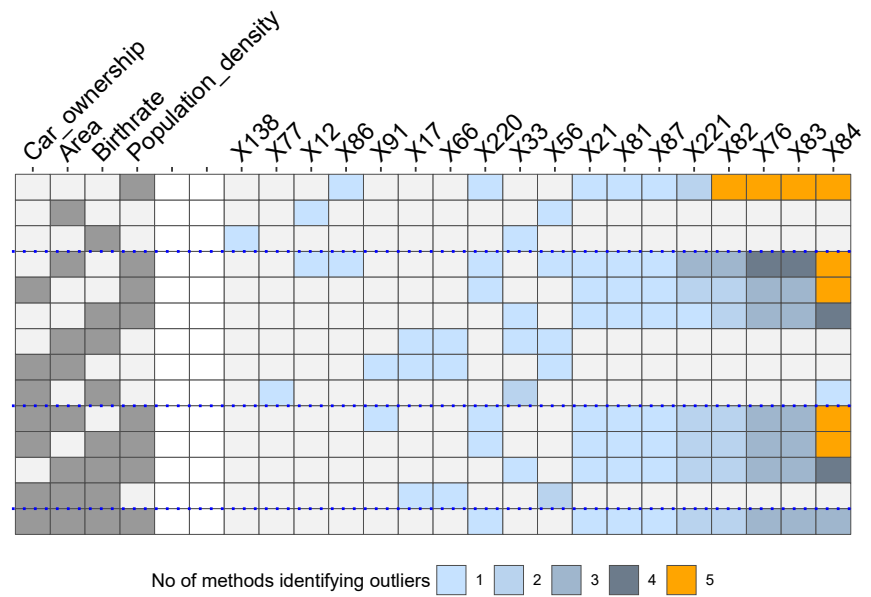

(a)

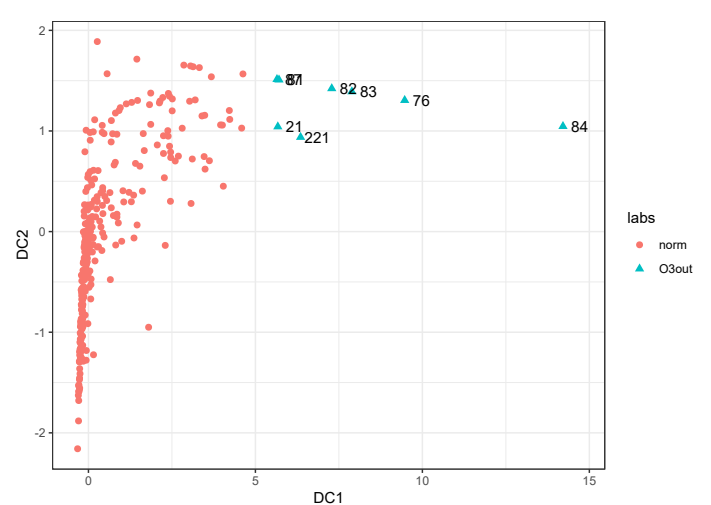

(b)

Figure 5: O3plot of the Election2005 dataset in Figure 5a and the first 2 DOBIN components in Figure $5 b$

Figure 5a shows the $\mathrm{O} 3$ plot of this dataset using all six outlier methods. The columns on the left indicate the variables, the columns on the right indicate the observations, the rows specify the axis parallel subspaces and the colours depict the number of methods that identify each observation in each subspace as an outlier. From this plot we see that observation $X 84$ is identified as an outlier by 5 methods in 5 subspaces, 4 methods in 2 subspaces, 3 methods in 1 subspace and by 1 method in 1 subspace. X84 is arguably the most outlying observation in this dataset. The observations $X 83, X 76, X 82$ are also identified as outliers by 5 methods in the dimension of population density. They are also identified as outliers by multiple methods in different subspaces. The layout of the $\mathrm{O} 3$ plot is such that the outlyingness of the observations increase to the right.

Figure $5 \mathrm{~b}$ shows the first 2 DOBIN components of the Election 2005 dataset. This is a projection of the dataset onto a two dimensional subspace spanned by the first 2 DOBIN vectors. Here we see the observation X84 (denoted by 84 on the plot) far away from the rest of the data with 
$X 76, X 83$ and $X 82$ somewhat detached from the rest. The observations $X 87$ an $X 81$ are almost at the same position in this 2-dimensional space. The observations $X 221$ and $X 21$ also appear a bit outside the boundary of other points. A simple KNN algorithm in the DC1-DC2 space gives the top 8 candidates as $X 84, X 76, X 83, X 82, X 221, X 81, X 87$, and $X 21$. Thus, the DC1-DC2 space accentuates the eight most outlying observations according to the $\mathrm{O} 3$ plot, while showing clearly that $\mathrm{X} 84$ is the most outlying observation.

The first DOBIN vector is of interest to us as the outliers deviate most in this direction. Equation (9) gives the first DOBIN vector, DC1, as a linear combination of the input variables.

$$
\mathrm{DC} 1=\left[\begin{array}{llll}
0.008 & 0.998 & 0.040 & -0.050
\end{array}\right]\left[\begin{array}{c}
\text { Area } \\
\text { Population Density } \\
\text { Birthrate } \\
\text { Car Ownership }
\end{array}\right]
$$

From equation (9) we see that Population Density is the main variable contributing to outliers in the DC1-DC2 space. The O3 plot supports this observation. If we look carefully at the O3 plot we see that X21 to X84 gets identified as outliers in all subspaces that contain Population Density. This insight gives us a better understanding of the dataset.

\subsection{Diamonds dataset}

The next example is taken from the O3Outliers R package (Unwin, 2019b) and uses the Diamonds dataset (Wickham, 2016). This dataset contains attributes of diamonds.

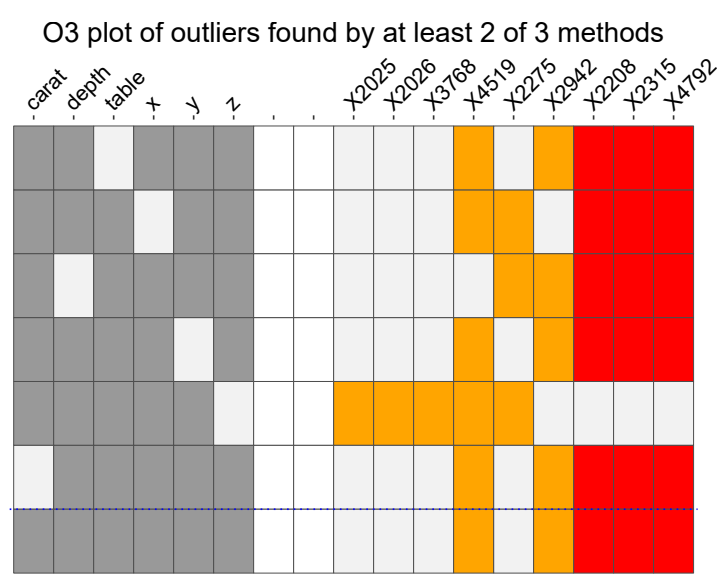

(a)

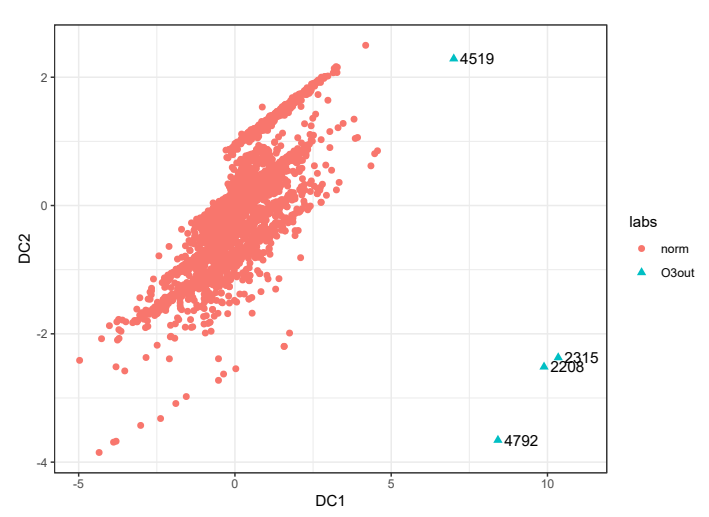

(b)

Figure 6: O3plot of the Diamonds dataset in Figure $5 a$ and the first 2 DOBIN components in Figure $5 b$. 
Figure 6a shows the O3 plot of this dataset using the three outlier methods HDoutliers, FastPCs and adjOutlyingness. We could not use all six methods on this dataset as the other three methods included in the OutliersO3 R package gave computational errors. Of the three methods used, all methods identified X4792, X2315 and X2208 as outliers in 6 subspaces including the full space. These 3 points appear in the DC1-DC2 space in the bottom right corner. In addition, the point X4519 (denoted by 4519 in the plot) appears in the top right corner in the DC1-DC2 space, away from other points. Comparing with the O3 plot, we see that X4519 is identified as an outlier by 2 methods in 6 subspaces. In keeping with the guidelines of the previous section, we use half of the DC components to evaluate a KNN algorithm. The top 4 candidates in this 3-dimensional DC space are X4792, X2315, X2208 and X4519 in that order.

To understand the first DOBIN vector, we look at its coefficients:

$$
\mathrm{DC} 1=\left[\begin{array}{llllll}
0.098 & -0.659 & 0.185 & 0.097 & 0.126 & -0.704
\end{array}\right]\left[\begin{array}{c}
\text { Carat } \\
\text { Depth } \\
\text { Table } \\
x \\
y \\
z
\end{array}\right]
$$

We see that the variables $z$ and Depth contribute more to outliers in the DC1-DC2 space as well as in the $\mathrm{O} 3$ plot.

\subsection{USArrests dataset}

The popular USArrests dataset is studied by Bailey \& Gatrell, 1995; Sarkar, 2008; Yamini, 2019 and many others. This dataset contains violent crime rates for the 50 US states in 1973 . The O3 plot using all six outlier detection methods and the DC1-DC2 space of this dataset are illustrated in Figure 7.

From Figure7a we see that X2 (corresponding to Alaska) is flagged as an outlier in 8 subspaces including the full space, by one or two outlier detection methods. The adjoining outlier X33 (North Carolina) is only flagged in 4 subspaces and the others in even smaller subspaces. Of the six outlier methods, only two identify Alaska as an outlier in certain subspaces. This also suggests that even though there is consensus between two methods, as only one third of the methods agree on this outlier, it may not be quite so outlying as the outliers in the previous examples. Indeed, this notion is validated by the DC1-DC2 plot. We see the observation X2 


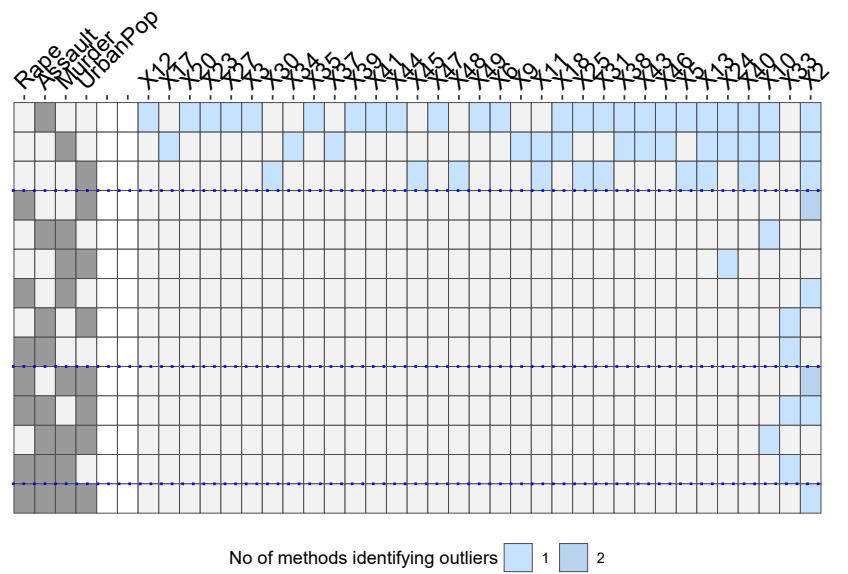

(a)

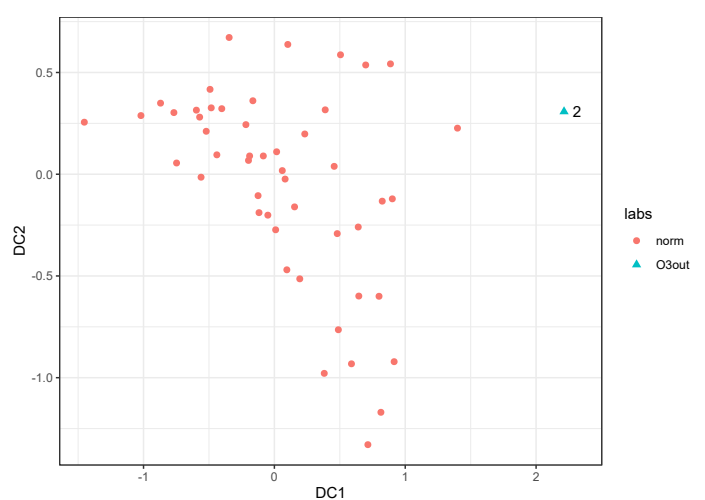

(b)

Figure 7: O3plot of the USArrests dataset in Figure 7a and the first 2 DOBIN components in Figure $7 b$.

(denoted by 2 in the plot) standing out from the rest, but not by a large margin. A KNN algorithm identifies this observation as having the highest KNN distance in DC1-DC2 space.

We look at the coefficients of the first DOBIN vector as X2 deviates in DC1:

$$
\mathrm{DC} 1=\left[\begin{array}{llll}
0.173 & 0.077 & -0.675 & 0.713
\end{array}\right]\left[\begin{array}{c}
\text { Murder } \\
\text { Assault } \\
\text { UrbanPop } \\
\text { Rape }
\end{array}\right]
$$

We see that the variables UrbanPop and Rape contribute more to the first DOBIN vector than the other variables. From equation (10), high values of DC1 indicate low percentages of urban population and high rape arrests per 100,000 residents. Therefore, we might expect Alaska to have higher rape arrests per urban capita. Indeed from Figure 8 we see that Alaska has the highest ratio of Rape/UrbanPop of all 50 states confirming the insight gleaned from the first DOBIN vector.

\subsection{Airquality dataset}

The Airquality dataset discussed in John et al., 1983 has measurements on air quality in New York city from May to September 1973. The O3 plot using all 6 outlier methods and the DC1-DC2 space of this dataset are shown in Figure 9.

The O3 plot in Figure 9a shows X117 as the only outlier identified in 6 subspaces by 2 methods. The associated DC1-DC2 space shows the observation X117 (denoted by 117 in the plot) away from the rest at the bottom right. Again, we see agreement between the O3 plot and DC1-DC2 


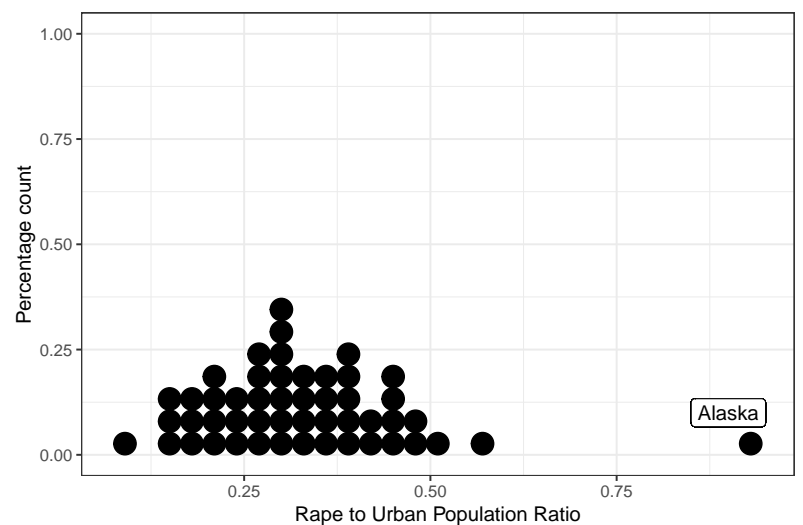

Figure 8: USArrests dataset - Rape to Urban population ratio values.

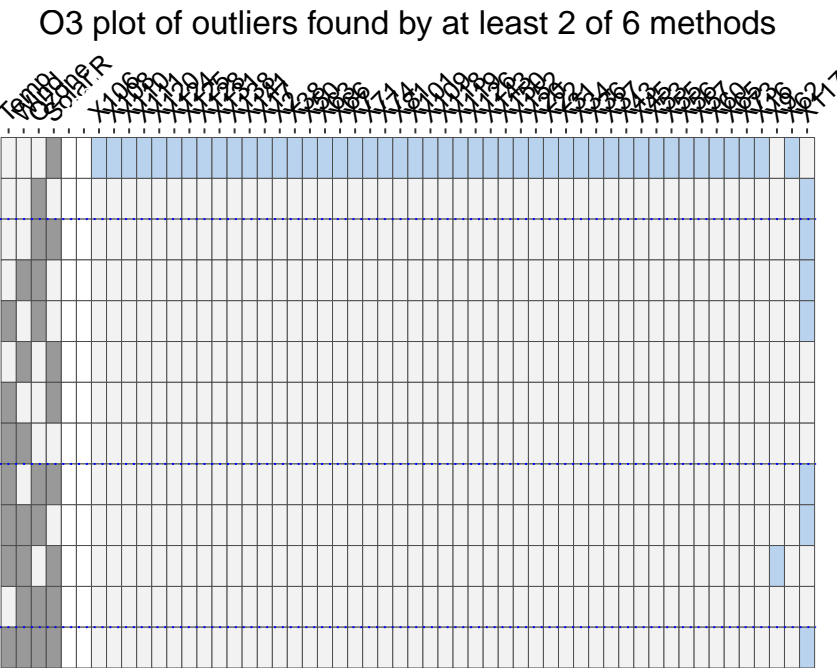

(a)

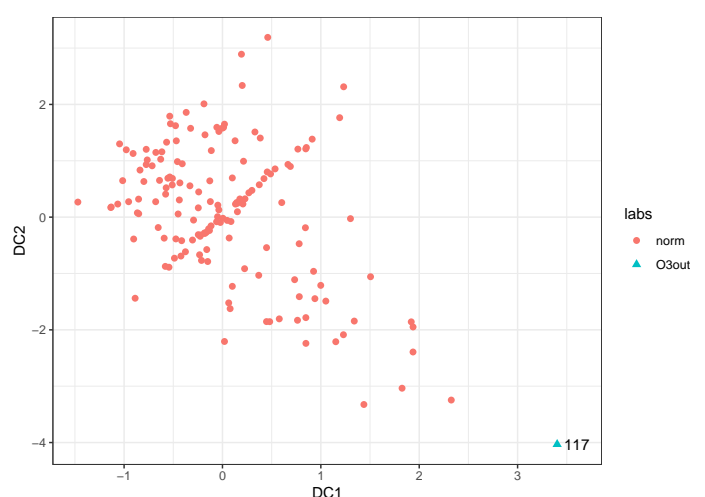

(b)

Figure 9: O3plot of the Airquality dataset in Figure 9a and the first 2 DOBIN components in Figure 9b.

space. The observation X117 was identified as an outlier only by a third of the outlier methods. This is corroborated in the DC1-DC2 plot by comparatively less outlyingness of observation X117 compared to outliers in the Diamonds dataset. Performing a KNN algorithm revealed the observation with the highest KNN distance as X117 in the DC1-DC2 space.

The first DOBIN vector has the following coefficients:

$$
\mathrm{DC} 1=\left[\begin{array}{llll}
0.842 & -0.078 & 0.512 & -0.151
\end{array}\right]\left[\begin{array}{c}
\text { Ozone } \\
\text { Solar.R } \\
\text { Wind } \\
\text { Temp }
\end{array}\right]
$$

From equation (11) we see that the observation X117 is an outlier due to high values of Ozone and Wind. 


\section{5 lesmis dataset}

The lesmis dataset (Vialaneix et al., 2019) contains the character coappearance network of characters in the novel Les Misérables by Victor Hugo. This dataset is stored as a graph, with vertices corresponding to characters and is shown in Figure 10. The character network in Les Misérables is also studied in Wilkinson, 2017 in the context of outlier detection. Here we conduct a similar study using the $\mathrm{O} 3$ plot and the DC1-DC2 space.

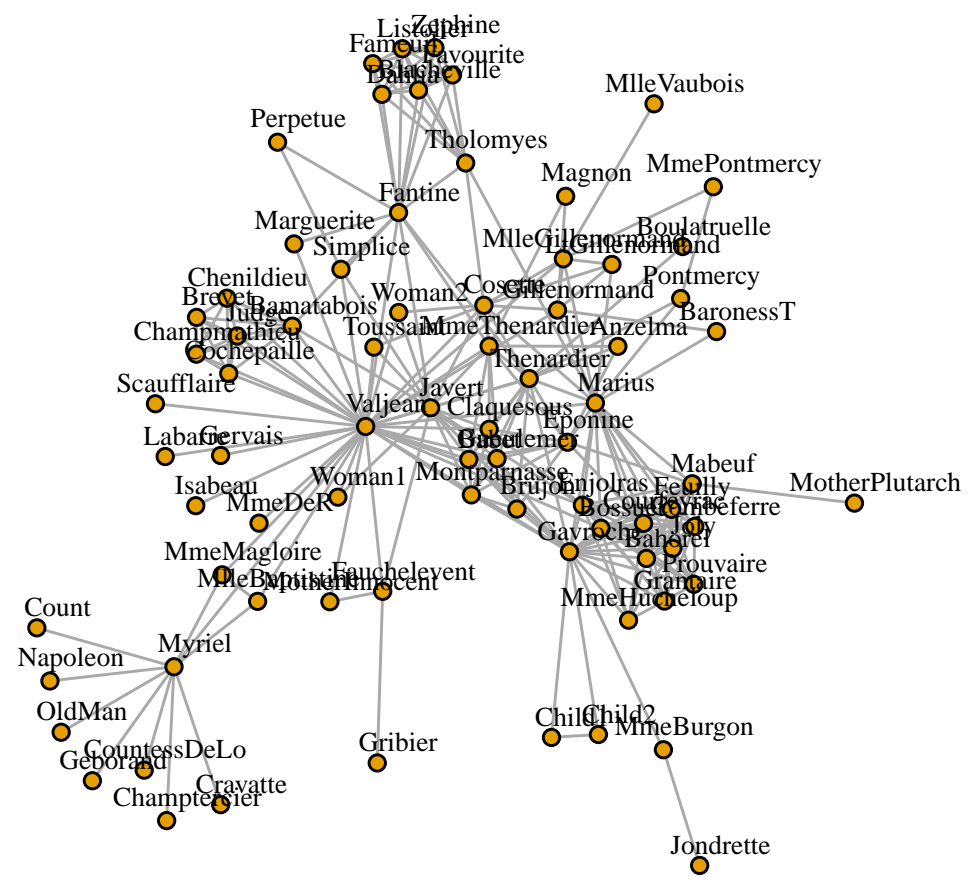

Figure 10: The lesmis dataset.

As in Wilkinson, 2017 we compute the graph-based features centrality, transitivity, closeness, betweenness, degree, average nearest neighbour degree and page rank for each character in the dataset. This transforms the original graph to a rectangular dataset of 77 observations and 7 variables, with each observation corresponding to a character in the novel. Figure 11a shows the O3 plot for the transformed lesmis dataset using the three methods HDoutliers, mvBACON and $\operatorname{covMCD}$. The other three outlier methods used in $\mathrm{O} 3$ plots gave computational errors and so could not be used. Figure 11b shows the DC1-DC2 space of the transformed lesmis dataset.

From the $\mathrm{O} 3$ plot it is evident that Valjean is the main outlier consistently identified by the three outlier methods in the vast majority of the subspaces. This is confirmed by the DC1-DC2 plot as Valjean appears far away from the rest of the characters in the top right corner. The next most outlying characters in the $\mathrm{O} 3$ plot are Myriel, Gavroche, Marius and Fantine. These characters also appear in the DC1-DC2 space somewhat detached from the rest of the characters, although 
O3 plot of outliers found by at least 2 of 3 methods

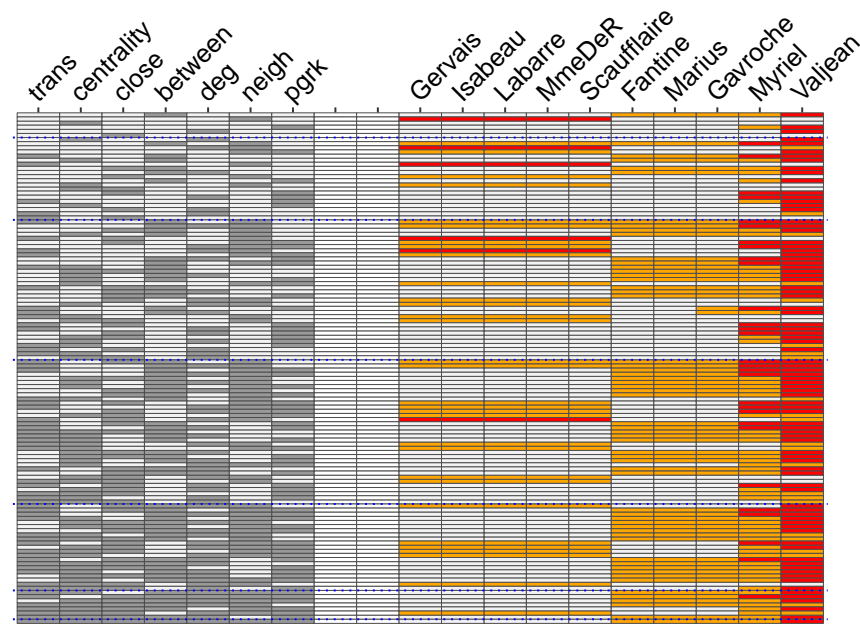

(a)

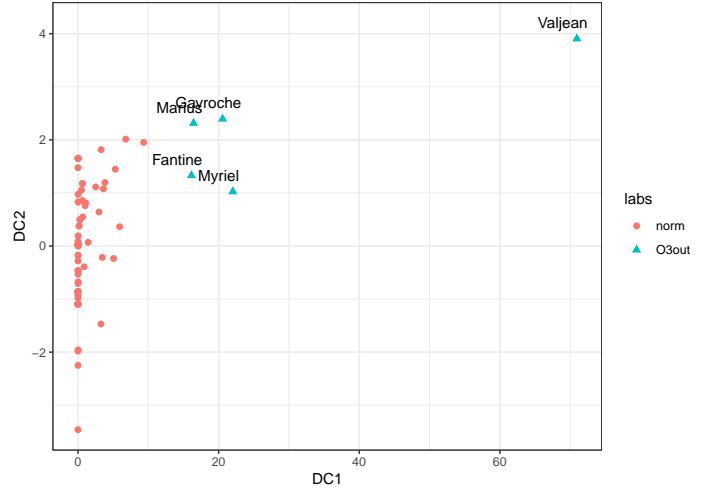

(b)

Figure 11: O3plot of the transformed lesmis dataset in Figure 11a and the first 2 DOBIN components in Figure $11 b$.

none so remarkably far away as Valjean. A KNN algorithm performed on the first half of the DC components identified Valjean, Myriel, Gavroche, Marius and Fantine as having the highest KNN distances.

As the first DOBIN vector is discriminatory, we look at its coefficients: The first DOBIN vector has the following coefficients:

$\mathrm{DC} 1=\left[\begin{array}{lllllll}0.0022 & -0.0001 & 0.0032 & 0.9999 & 0.0029 & 0.0025 & 0.0057\end{array}\right]\left[\begin{array}{c}\text { Centrality } \\ \text { Transitivity } \\ \text { Closeness } \\ \text { Betweenness } \\ \text { Degree } \\ \text { Avg. Neighbour Degree } \\ \text { Page Rank }\end{array}\right]$.

From equation (12) we see that Betweenness is the main feature that makes Valjean an outlier in the DC1-DC2 space. The property Betweenness is defined as the number of shortest paths going through a vertex (Csardi \& Nepusz, 2006). Thus Valjean becomes a node in many shortest paths in the character network making him the main outlier. 


\subsection{Classics from Gutenberg}

This example is on text analysis of 22 classics downloaded from the Gutenberg project (Project Gutenberg n.d.) and is similar to the example in Wilkinson, 2017. We consider the novels Alice in Wonderland, Anna Karenina, Bleak House, Emma, Frankenstein, Gullivers Travels, Jude the Obscure, Lord Jim, Mansfield Park, Middlemarch, Moby Dick, Northanger Abbey, Persuasion, Pride and Prejudice, Sense and Sensibility, Silas Marner, Sons and Lovers, The Life and Opinions of Tristram Shandy, Wizard of $\mathrm{Oz}$, Ulysses, Vanity Fair and War and Peace in our analysis.

We strip each novel into words and obtain the collection of words used and their relative frequencies. This set of words need to be cleaned before computing any useful features. We use the R package tidytext (Silge \& Robinson, 2016) for this task. As the first 'cleaning' step, we eliminate the stop words such as the, $a$, an and in from our collection. We also remove numbers from our word collection. Next we use a stemming procedure to reduce words to their word stem. For example the words run and running have the same word stem. This process gives us a cleaned set of words for each novel. We compute the tf-idf (term frequency-inverse document frequency) measure on this collection. The tf-idf statistic measures how important a word is to a document when considering a collection of documents. Consequently we end up with a rectangular dataset of 22 observations and 39, 624 variables, where each observation is a novel and each variable is the tf-idf statistic of a word.

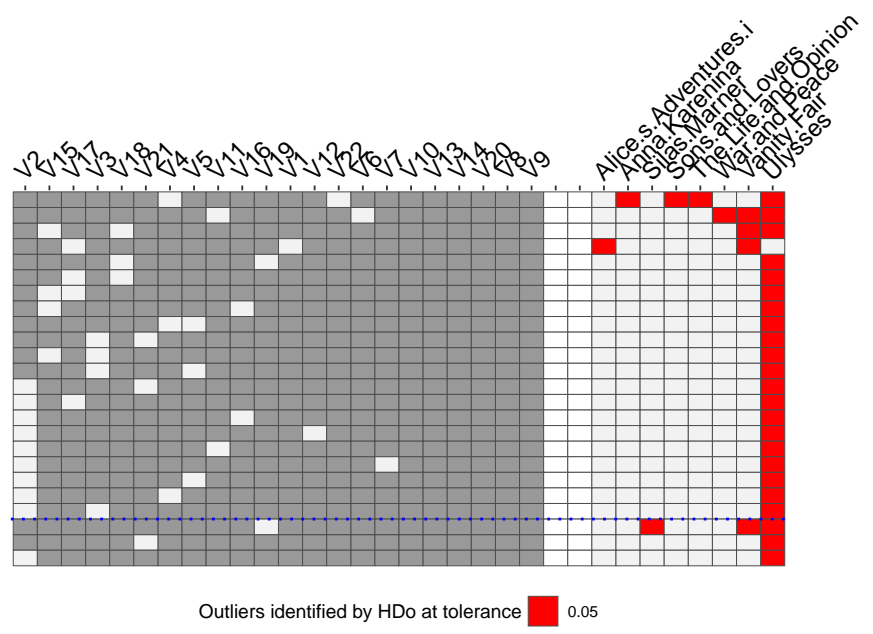

(a)

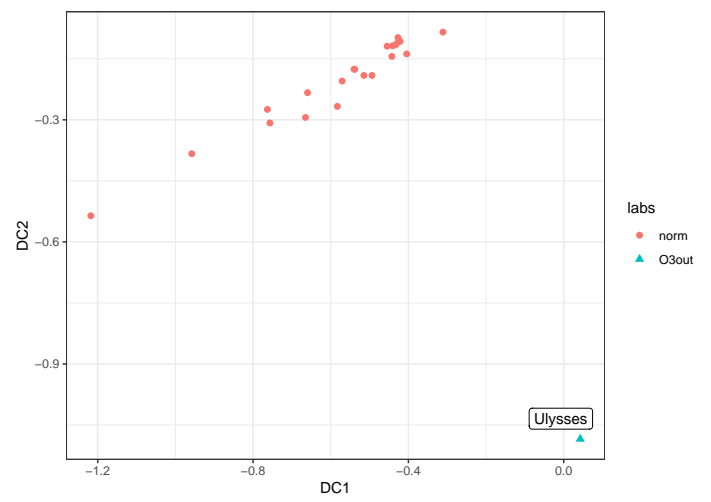

(b)

Figure 12: O3plot of 22 Gutenberg classics in Figure 12a and the first 2 DOBIN components in Figure $12 b$.

However, 22 vectors in $\mathbb{R}^{39,624}$ can only span a subspace of 22 dimensions or less. Therefore, we compute this subspace and the respective coordinates of the observations using PCA. We use all 
the principal components so that we're performing a change of basis and not a projection. As a result we obtain a $22 \times 22$ matrix, where each row is an observation corresponding to a novel and each column is a linear combination of $39,624 \mathrm{tf}-\mathrm{idf}$ statistics. The resulting O3 plot using HDoutliers and the corresponding DC1-DC2 space is illustrated in Figure 12. Again we see the O3 plot and the DC1-DC2 space agreeing on the most outlying novel Ulysses confirming the findings of Wilkinson, 2017. Performing a KNN algorithm yielded Ulysses as the observation with the highest KNN distance.

The novel Ulysses stands out in the DC1-DC2 space mainly because of its second DC component. However, we do not explore the coefficients of this vector as each coefficient is a linear combination of 39, 624 variables, making it difficult to gain insights.

This section substantiated DOBIN's ability as an outlier visualization tool. In each example we saw the outliers projected onto a 2D space spanned by the first two DOBIN vectors, and their legitimacy was endorsed by the $\mathrm{O} 3$ plots. Furthermore, we gained insights about the nature of outlyingness in data by inspecting DOBIN vectors.

\section{Results on a data repository}

In this section we work with a data repository of 12,642 datasets (Kandanaarachchi et al., 2019), generated from approximately 200 source datasets. Each dataset has labeled outliers and originally was used for classification purposes. The present datasets are modified from its original form by downsampling minority class observations, converting categorical attributes to numerical values, removing missing data and creating several variants from each classification dataset. More details on dataset preparation can be found in Kandanaarachchi et al., 2018. This collection also includes datasets from Campos et al., 2016.

A data point that is clearly an outlier in a subspace may be difficult to detect as an outlier in the full space, particularly when other variables drown the subspace outlier (Zimek, Schubert \& Kriegel, 2012). For example in Figure 12a, the novel Alice in Wonderland is detected as an outlier in the fourth subspace in the $\mathrm{O} 3$ plot, but not in others. Conversely, when testing algorithms for outlier detection, we need to consider data where some variables contribute noise that mask outliers.

Therefore, to make these datasets suitable for testing our outlier detection algorithms, we "fatten" each dataset by adding 20 noise variables distributed normally, i.e. $\sim \mathcal{N}(0,1)$. We use these 'fattened' datasets in our experiment. 
Similar to Section 3, for each dataset we compare the results of three outlier methods LOF, KNN and iForest using the area under the ROC curve (AUC), on three different coordinate systems, namely the full set of coordinates, the first half of the DOBIN components and the first half of the Principal Components. That is, for a dataset with $p$ variables and $N$ observations, we consider $\lfloor\min (N / 2, p / 2)\rfloor$ DOBIN and PC components.

Furthermore, we need to select appropriate tools to present results of 12,642 datasets across three methods and three coordinate systems. The ability to delve into each dataset and obtain insights is no longer feasible with a large repository. In addition, we want to ascertain whether DOBIN coordinates improve the performance of the three outlier detection methods compared to the other two coordinate systems. Therefore, we investigate each outlier detection method separately. For each outlier method, we compare the performance of the three coordinate systems using a Friedman test adjusting for the dataset variants. To adjust for the dataset variants, we take the median performance for each dataset source and each coordinate system. If the Friedman test is significant, then we perform a Nemenyi test, so that we can rank the coordinate systems according to performance. For convenience we use the shortened names $1 / 2$ DOBIN for using the first half of DOBIN components, 1/2 PCA for using the first half of Principal Components and 'All Vars' for using all variables in the remainder of the section.

\subsection{Significant tests}

Figure 13 gives the results of the three coordinate systems on outlier detection method LOF. A Friedman test gave the $p$-value $1.986 \times 10^{-5}$, and Figure 13a shows the associated Nemenyi plot for this data. This plot shows the ranks of the coordinate systems, with lower ranks indicating better performance. The blue squares highlight methods which are not significantly different from each other. From Figure 13a we see that the best coordinates for LOF are 1/2 DOBIN, followed by 1/2 PCA and All Vars, with 1/2 PCA and All Vars not significantly different from each other. Figure 13b shows the boxplots of performance differences between the three coordinate systems. Positive values in AUC difference between coordinates indicate an improvement of one coordinate system over the other. We see that $1 / 2$ DOBIN $-1 / 2$ PCA and 1/2 DOBIN - All Vars have positive median AUC differences unlike 1/2 PCA - All Vars.

Figure 14 shows the results of the three coordinate systems on the outlier detection method KNN. We obtained the $p$-value $1.341 \times 10^{-8}$ from the Friedman test. Again, from Figure 14a we see that $1 / 2$ DOBIN outperforms All Vars and 1/2 PCA, which is corroborated by the boxplots 


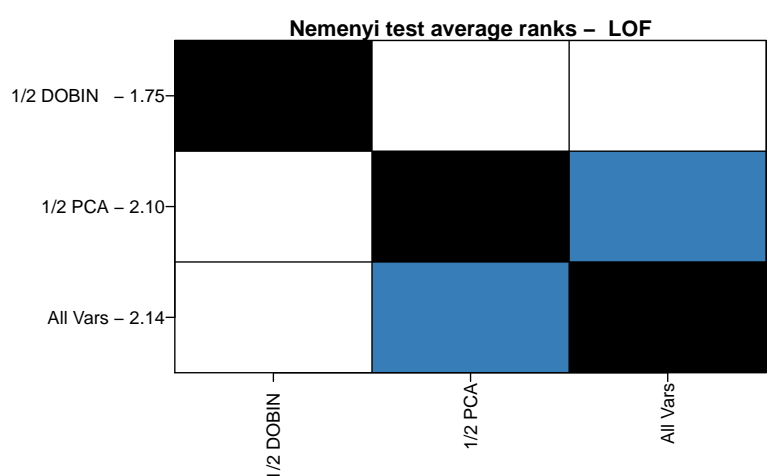

(a)

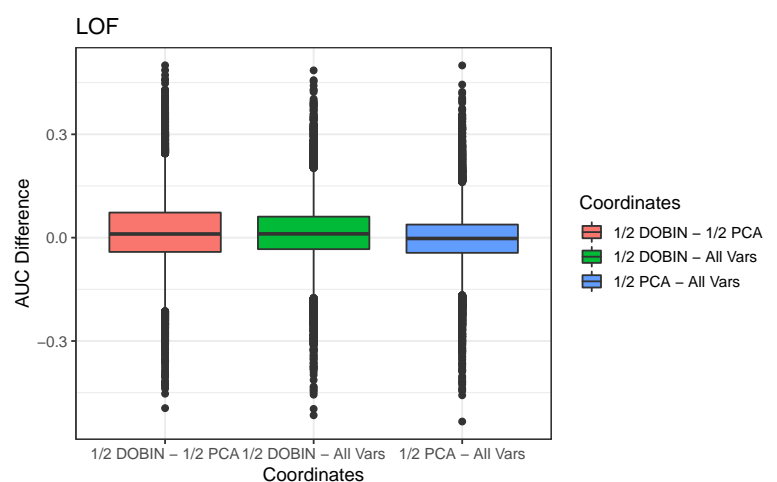

(b)

Figure 13: Nemenyi plot of LOF results and the boxplots of performance differences in Figures $13 a$ and $13 b$.

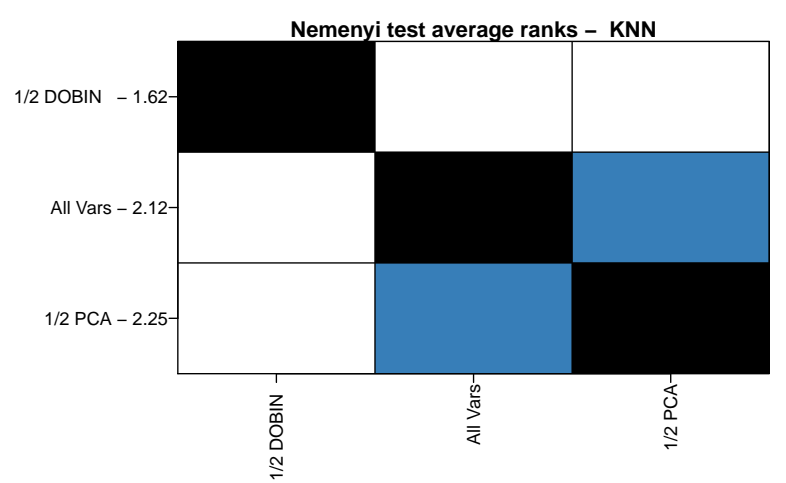

(a)

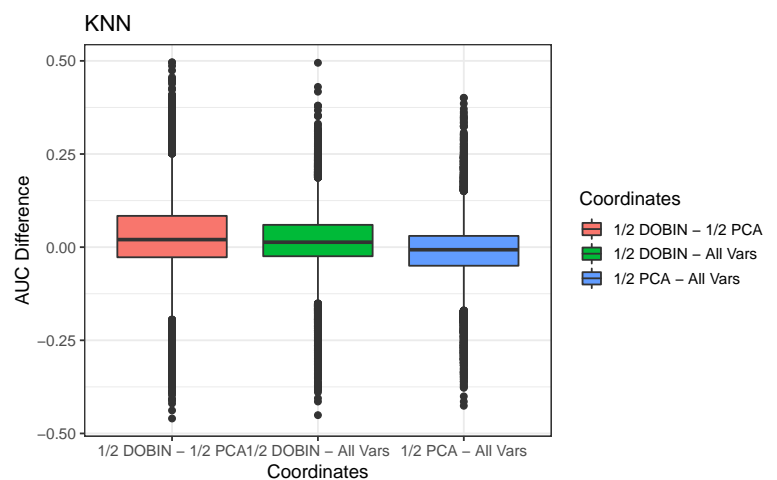

(b)

Figure 14: Nemenyi plot of KNN results and the boxplots of performance differences in Figures $14 a$ and $14 b$.

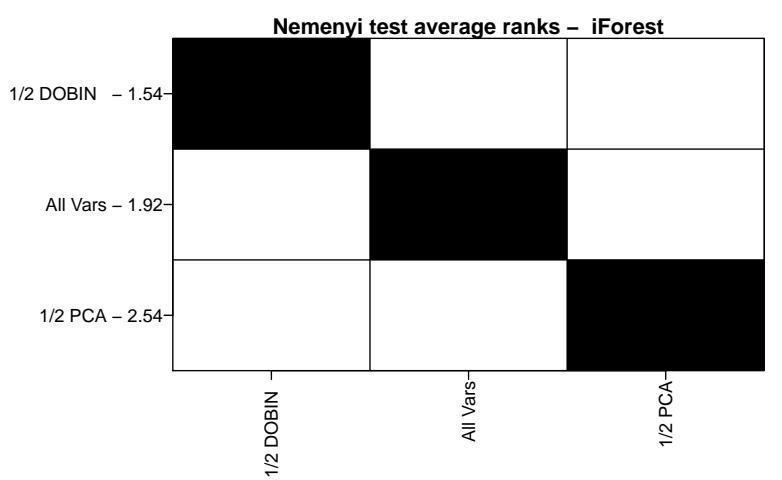

(a)

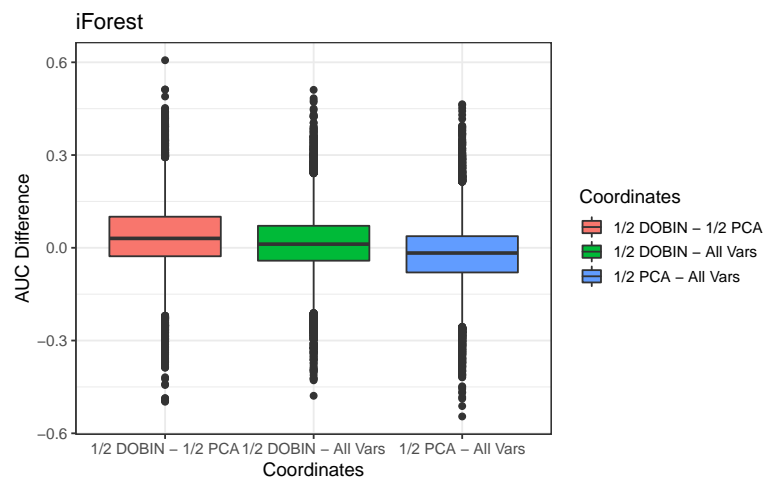

(b)

Figure 15: Nemenyi plot of iForest results and the boxplots of performance differences in Figures $15 a$ and $15 b$. 
in Figure 14b. Even though All Vars is ranked second, it is not significantly different from 1/2 PCA for KNN.

Similar performances are obtained for iForest, which is illustrated in Figure 15. A Friedman test conducted on the data gave a $p$-value less than $2.2 \times 10^{-16}$. The Nemenyi plot shows that while $1 / 2$ DOBIN performs the best, all three methods are significantly different from each other, with All Vars outperforming 1/2 PCA. This is supported from the boxplots in Figure 15b, with 1/2 DOBIN - 1/2 PCA and 1/2 DOBIN - All Vars having positive median AUC differences and 1/2 PCA - All Vars having a negative one.

We see that $1 / 2$ DOBIN performs best for all three outlier detection methods and is significantly better than 1/2 PCA and All Vars. This is evidence that DOBIN is better as a dimension reduction tool for outier detection compared to PCA and the original variables. We attribute this success to DOBIN's firm mathematical foundations.

\section{Conclusion}

Dimension reduction for outlier detection is a topic that has not received much attention in the literature. One way to achieve this is to construct a basis that empowers outlier detection. In this paper, we present DOBIN: a Distance based Outlier BasIs using Neighbours, which strives to accomplish this goal. We used DOBIN on an extensive data repository of real world datasets and showed that DOBIN basis improves performance of the outlier detection methods LOF, KNN and iForest. Using half of the DOBIN components we outperformed PCA and the standard basis as coordinate systems for these three outlier detection methods.

In addition, we conducted a visual analysis of outliers using DOBIN on six datasets from diverse sources, which included a character graph from the novel Les Misérables and 22 classics from Gutenberg website. We projected the data onto a 2D space spanned by the first two DOBIN vectors, and saw that outliers identified by many methods in $\mathrm{O} 3$ plots were indeed far away from the rest of the data. We gained insights about the outliers in these datasets, and identified the variables that contributed to make them outliers.

Currently DOBIN is sensitive to rotations in the input space. In future work we plan to investigate avenues that can contribute to a rotation invariant version of DOBIN. One possibility, for example, would be to first perform a PCA decomposition followed by DOBIN. We 
would gain rotation-invariance from such a version, but we would potentially lose some interpretablity of the results. The $\mathrm{R}$ package dobin contains the implementation and is available at https://github.com/sevvandi/dobin for download.

\section{Supplementary Material}

R package dobin: This package contains the DOBIN basis construction.

Datasets: Datasets discussed in Section 5 are available at Kandanaarachchi et al., 2019. The character network from the novel Les Misérables was taken from the R package SOMbrero (Vialaneix et al., 2019). The book vectors dataset used in Section 4.6 is available at the github repository https://github.com/sevvandi/Outlier-Basis.

Scripts: The script Figures_For_Paper_1.R contains the R code used to conduct experiments and produce graphs in Section 3. The script Figures_For_Paper_2.R contains the R code used in Section 4. And finally, the script Figures_For_Paper_3.R contains the R code used to produce the graphs in Section 5. The original computation on the data repository detailed in Section 5 was conducted using the MonARCH HPC Cluster. The results of this computation are available at the github repository https://github.com/sevvandi/Outlier-Basis.

Other R-packages: In addition to the $\mathrm{R}$ package dobin we have also used the $\mathrm{R}$ packages OutliersO3 (Unwin, 2019b), isolationForest (Liu, 2009), DMwR (Torgo, 2010), pROC (Robin et al., 2011), ggplot2 (Wickham, 2016), mbgraphic (Grimm, 2019), gridExtra (Auguie, 2017), tsutils (Kourentzes, 2019), tidyverse (Wickham, 2017) and tidyr (Wickham \& Henry, 2018).

\section{Acknowledgements}

Funding was provided by the Australian Research Council through the Linkage Project LP160101885. This research was also supported by the Monash eResearch Centre and eSolutionsResearch Support Services through the MonARCH HPC Cluster.

\section{References}

Aggarwal, CC \& PS Yu (2001). Outlier detection for high dimensional data. ACM Sigmod Record 30(2), 37-46.

Auguie, B (2017). gridExtra: Miscellaneous Functions for "Grid" Graphics. R package version 2.3. https: //CRAN . R-project .org/package=gridExtra. 
Bailey, TC \& AC Gatrell (1995). Interactive spatial data analysis. Vol. 413. Longman Scientific \& Technical Essex.

Billor, N, AS Hadi \& PF Velleman (2000). BACON: blocked adaptive computationally efficient outlier nominators. Computational statistics $\mathcal{E}$ data analysis 34(3), 279-298.

Breunig, MM, HP Kriegel, RT Ng \& J Sander (2000). LOF: identifying density-based local outliers. ACM Sigmod Record 29(2), 93-104.

Brys, G, M Hubert \& P Rousseeuw (2005). A robustification of independent component analysis. Journal of Chemometrics: A Journal of the Chemometrics Society 19(5-7), 364-375.

Campos, GO, A Zimek, J Sander, RJ Campello, B Micenková, E Schubert, I Assent \& ME Houle (2016). On the evaluation of unsupervised outlier detection: measures, datasets, and an empirical study. Data Mining and Knowledge Discovery 30(4), 891-927.

Csardi, G \& T Nepusz (2006). The igraph software package for complex network research. InterJournal Complex Systems, 1695. http: //igraph . org.

Goldstein, M \& S Uchida (2016). A comparative evaluation of unsupervised anomaly detection algorithms for multivariate data. PloS one 11(4), e0152173.

Grimm, K (2019). mbgraphic: Measure Based Graphic Selection. R package version 1.0.1. https : //CRAN.R-project.org/package=mbgraphic.

Hyndman, RJ, E Wang \& N Laptev (2015). Large-scale unusual time series detection. In: 2015 IEEE international conference on data mining workshop (ICDMW). IEEE, pp.1616-1619.

John, C, W Cleveland, B Kleiner \& P Tukey (1983). Graphical methods for data analysis. Pacific Grove: Wadsworth, 158-62.

Kandanaarachchi, S (2019). dobin: Dimension Reduction for Outlier Detection. R package version 0.0.0.9000. https://github.com/sevvandi/dobin.

Kandanaarachchi, S, M Muñoz Acosta, K Smith-Miles \& R Hyndman (Feb. 2019). Datasets for outlier detection. https: //monash . figshare . com/articles/Datasets\%5C_12338\%5C_zip/ $7705127 / 4$.

Kandanaarachchi, S, MA Munoz, RJ Hyndman, K Smith-Miles, et al. (2018). On normalization and algorithm selection for unsupervised outlier detection. pre-print. http : / / bit . ly / normout.

Keller, F, E Muller \& K Bohm (2012). HiCS: High contrast subspaces for density-based outlier ranking. In: 2012 IEEE 28th international conference on data engineering. IEEE, pp.1037-1048.

Kourentzes, N (2019). tsutils: Time Series Exploration, Modelling and Forecasting. R package version 0.9.0. https://CRAN.R-project.org/package=tsutils. 
Kriegel, HP, P Kröger, E Schubert \& A Zimek (2009). LoOP: local outlier probabilities. In: Proceedings of the 18th ACM conference on Information and knowledge management. ACM, pp.16491652.

Liu, FT (2009). IsolationForest: Isolation Forest. R package version 0.0-26/r4. https ://R-Forge. Rproject.org/projects/iforest/.

Liu, FT, KM Ting \& ZH Zhou (2008). Isolation forest. In: 2008 Eighth IEEE International Conference on Data Mining. IEEE, pp.413-422.

Project Gutenberg (n.d.). http://www . gutenberg . org. Accessed: 2019-08-30.

Ramaswamy, S, R Rastogi \& K Shim (2000). Efficient algorithms for mining outliers from large data sets. ACM Sigmod Record 29(2), 427-438.

Robin, X, N Turck, A Hainard, N Tiberti, F Lisacek, JC Sanchez \& M Müller (2011). pROC: an open-source package for $\mathrm{R}$ and $\mathrm{S}+$ to analyze and compare ROC curves. BMC Bioinformatics $12,77$.

Rousseeuw, PJ \& WVD Bossche (2018). Detecting deviating data cells. Technometrics 60(2), 135145.

Rousseeuw, PJ \& KV Driessen (1999). A fast algorithm for the minimum covariance determinant estimator. Technometrics 41(3), 212-223.

Rousseeuw, PJ \& AM Leroy (2005). Robust regression and outlier detection. John wiley \& sons.

Sarkar, D (2008). Labels and legends. Lattice: Multivariate Data Visualization with R, 151-163.

Silge, J \& D Robinson (2016). tidytext: Text Mining and Analysis Using Tidy Data Principles in R. JOSS 1(3). http://dx.doi.org/10.21105/joss. 00037.

Talagala, PD, RJ Hyndman, K Smith-Miles, S Kandanaarachchi \& MA Muñoz (2019). Anomaly detection in streaming nonstationary temporal data. Journal of Computational and Graphical Statistics. just-accepted, 1-28.

Torgo, L (2010). Data Mining with R, learning with case studies. Chapman and Hall/CRC. http: //www.dcc.fc.up.pt/ Itorgo/DataMiningWithR.

Unwin, A (2019a). Multivariate outliers and the O3 Plot. Journal of Computational and Graphical Statistics, 1-11.

Unwin, A (2019b). OutliersO3: Draws Overview of Outliers (O3) Plots. R package version 0.6.2. https : //CRAN. R-project .org/package=0utliers03.

Vakili, K \& E Schmitt (2014). Finding multivariate outliers with FastPCS. Computational Statistics $\mathcal{E}$ Data Analysis 69, 54-66. 
Vialaneix, N, J Mariette, M Olteanu, F Rossi, L Bendhaiba \& J Bolaert (2019). SOMbrero: SOM Bound to Realize Euclidean and Relational Outputs. R package version 1.2-4.

Wickham, H (2016). ggplot2: Elegant Graphics for Data Analysis. Springer-Verlag New York. http: //ggplot2.org.

Wickham, H (2017). tidyverse: Easily Install and Load the 'Tidyverse'. R package version 1.2.1. https://CRAN.R-project.org/package=tidyverse.

Wickham, H \& L Henry (2018). tidyr: Easily Tidy Data with 'spread()' and 'gather()' Functions. R package version 0.8.2. https ://CRAN . R-project . org/package=tidyr.

Wilkinson, L (2017). Visualizing big data outliers through distributed aggregation. IEEE transactions on visualization and computer graphics 24(1), 256-266.

Yamini, MPC (2019). A VIOLENT CRIME ANALYSIS USING FUZZY C-MEANS CLUSTERING APPROACH. ICTACT Journal on Soft Computing 9(3), 1939-1944.

Zimek, A, E Schubert \& HP Kriegel (2012). A survey on unsupervised outlier detection in high-dimensional numerical data. Statistical Analysis and Data Mining: The ASA Data Science Journal 5(5), 363-387. 